\title{
The Prevalence and Predictors of Low-Cost Generic Program Use in the Pediatric Population
}

\author{
Nathan James Pauly $^{1} \cdot$ Jeffery Charles Talbert $^{1} \cdot$ Joshua David Brown $^{1}$
}

Published online: 1 December 2015

(C) The Author(s) 2015. This article is published with open access at Springerlink.com

\begin{abstract}
Background Low-cost generic drug programs (LCGPs) increase the accessibility and affordability in the USA of prescription medication that can treat many common pediatric conditions. No studies have assessed the prevalence and predictors of LCGP use in the pediatric population, analyzed trends in LCGP use since their implementation, or analyzed which medications are most commonly purchased for children through LCGPs.

Objectives Our objective was to determine the prevalence of LCGP use in the USA during the period 2007-2012 and to assess predictors of LCGP use in a nationally representative sample of children and adolescents.

Methods We used cross-sectional data from the 2007-2012 Medical Expenditure Panel Survey (MEPS) and classified each prescription fill as an LCGP or nonLCGP fill. We assessed the proportions of LCGP fills and LCGP users each year from 2007 to 2012 and compared users and non-users during the latest available study cohort (2011-2012) using chi-squared and $t$-tests for users. We used multivariable logistic regression to identify factors associated with LCGP use in the most recent MEPS panel. Results Of 2754 children meeting all inclusion criteria, $23.7 \%$ were classified as LCGP users, representing over 10 million adolescent LCGP users over the 2011-2012 period. LCGP users were significantly more likely to be female, privately insured, White, residing in urban areas, lacking prescription drug coverage, and in a higher income bracket than non-users. Significant predictors of LCGP use included
\end{abstract}

Joshua David Brown

josh.brown@uky.edu

1 Institute for Pharmaceutical Outcomes and Policy (IPOP), University of Kentucky College of Pharmacy, 789 S.

Limestone, Lexington, KY 40536, USA age, prescription drug coverage, insurance type, race, region of residence, and number of unique medications used.

Conclusions IWhile one in four children use LCGPs, certain subgroups that may benefit the most from the programs are using them at a lower rate, and use of these programs has important effects on medication utilization quality assurance and research.

\section{Key Points}

Low-cost generic programs (LCGPs) increase access to medications, especially for those with lower income and no insurance, but they introduce the chance of exposure misclassification if medication use is unobserved, with implications for patients and health systems for quality assurance and research.

In the US pediatric population, $23.7 \%$ used at least one medication filled via an LCGP. However, uninsured children were half as likely to use LCGPs as children with private insurance.

Roughly $6 \%$ of all medication fills for children were obtained through LCGPs, including $>10 \%$ of fills for antidepressants, antibiotics, and cardiovascular medications with potentially serious adverse drug effects.

\section{Introduction}

Low-cost generic drug programs (LCGPs) first appeared in the USA in mid-2006, with Kmart providing 90 days of certain generics for $\$ \mathrm{US} 15$; this was shortly followed by 
Wal-Mart's \$US4 program [1]. Generic discount programs are now in place at almost all major pharmacy chains, including eight of the top ten largest chain pharmacies in the nation, and include one-third of the top 100 generics used by Americans by volume [1-3]. Medications available through LCGPs can be used to treat many common medical conditions experienced by children, including infections, asthma, allergies, and digestive disorders [4-6].

LCGPs have increased the accessibility and affordability of medications, especially for Americans living in poverty or without prescription insurance [2]. Given the low costs of these medications, it is often cheaper for individuals to purchase prescription medications out of pocket via LCGPs than to pay the \$US10-20 copay to receive medication through a prescription benefit [2, 7]. A 2011 study estimated potential savings from individuals purchasing medications through LCGPs instead of their insurance programs and determined that Americans could have saved over \$US1 billion in 2007 alone [8].

Despite this potential for tremendous cost savings, there is a relative dearth of literature assessing the prevalence of LCGP use in a nationally representative population. In 2008, over 70 million Americans were estimated to have used an LCGP to obtain a prescription medication-a figure that has likely expanded as the number and popularity of these programs has increased [1, 6, 9]. In a 2008 selfreported survey, one-third of adults and one-quarter of children without insurance coverage reported using these programs, and parents reported use of these programs for 14-23\% of children with private or public insurance [9]. However, given that self-reported surveys are particularly prone to recall and selection bias, it is difficult to ascertain whether these estimates accurately represent LCGP use in the pediatric and adolescent populations.

Use of LCGPs has important ramifications for health services research reliant on administrative claims data. Medication paid for out of pocket is a known source of medication exposure misclassification in administrative claims, and LCGPs are a common source of out-of-pocket payments [2, 7]. When individuals fill a medication through an LCGP, the dispensing pharmacist has no incentive to file a claim for the medication with the individual's insurer, meaning the medication use will be unobserved in the pharmaceutical claims data. If children with different forms of insurance-e.g., public, private, or uninsured-use LCGPs at different rates, it may lead to exposure misclassification in administrative claims sources. Given the expansion of insurance coverage under the Affordable Care Act (ACA), it is more important than ever to estimate how often individuals with different forms of insurance use LCGPs and how this use may impact the potential for differential exposure misclassification in claims data [10].
Few studies have assessed the demographic and clinical characteristics of LCGP users and non-users, and none to our knowledge have assessed LCGP use specifically in a pediatric population. Understanding the factors that influence which individuals currently utilize these programs is a crucial first step in increasing use amongst patients that have the most to gain from LCGPs and quantifying the potential effects that LCGP use may have on the healthcare system. This cross-sectional study had four objectives: (1) estimate the prevalence and predictors of LCGP use in a nationally representative pediatric population; (2) analyze trends amongst children in LCGP use since they were implemented in 2006; (3) determine which medications are most commonly purchased for children through LCGPs; and (4) assess the potential for exposure misclassification due to LCGP use.

\section{Methods}

\subsection{Data Source}

This study utilized public use data from the Medical Expenditure Panel Survey (MEPS) for the years 2007-2012. MEPS is a nationally representative survey of civilian, non-institutionalized individuals in the USA and includes information on demographics, healthcare utilization, medical conditions, and prescription medication use. MEPS uses an overlapping panel design with a new cohort ('panel') added each year that participates in the survey for up to 2 years. Data are collected in five rounds throughout a panel's 2 years of participation. Medication use is captured at the pharmacy level and includes all prescriptions obtained, irrespective of whether an insurance claim was submitted. Survey sampling and response weights are included so that population estimates may be obtained.

\subsection{Study Subjects and Design}

We used a cross-sectional study design to compare differences between LCGP users and non-users in the 2011-2012 MEPS panel. In a separate descriptive analysis, rates of LCGP use from the 2007-2012 data were quantified to assess trends in the proportions of LCGP fills and LCGP users over these years. These years were chosen because 2007 was the first full year in which LCGPs were available and 2012 is the most recent year of data available from MEPS. Both analyses had the same inclusion criteria, which required that individuals were aged 0-17 years, participated in all five rounds of data collection, and reported using at least one prescription medication during their 2-year panel period. The 2011-2012 data panel was chosen for the cross-sectional analysis as it was the most 
recent data panel available and provided the most up-todate analysis of LCGP use in the pediatric population. Study methods for inclusion of subjects, classification of LCGP fills, and classification as users and non-users were consistent across both the cross-sectional and the trend analysis.

Pharmaceutical utilization was assessed at the individual level for each year of the study period. Pharmaceutical data in MEPS include drug name, National Drug Code (NDC), MEPS round supplied, strength, quantity dispensed, and days supplied. Each prescription fill included in the MEPS dataset also includes information regarding the amount paid by the individual out of pocket and the amount contributed by other sources.

\subsection{Use of Low-Cost Generic Programs (LCGP)}

Four stipulations were used to define LCGP use: (1) the total cost of the drug was paid out of pocket (i.e., paid completely in cash by the customer); (2) the cost of the drug exactly matched the cost of an LCGP drug as reported by pharmacies (e.g., \$US4 cash payment); (3) the medication was available through an LCGP from a major chain pharmacy from 2007 to 2012; and (4) oral medication fills were dispensed for 30- or 90-day supplies of medications, with the exception of anti-infectives, contraceptives, and steroids, which were allowed to vary given the different days of supply typically dispensed for these classes. LCGP use was coded at the person level as a binary dependent variable for any use during the study period and at the medication level for each medication fill. Pharmaceutical utilization was determined for medications available from LCGPs based on Multum Lexicon classification systems (Cerner Multum $^{\mathrm{TM}}$, Denver, CO, USA). We calculated the proportion of fills of each medication or medication class obtained through an LCGP.

\subsection{Subject Characteristics}

Cohort demographics and characteristics of interest included age, race, sex, family income level, insurance type, prescription drug coverage, medical conditions, and number of prescriptions filled. For comparison, the cohort was stratified by age categories: 0-4, 5-8, 9-12, and 13-17 years. To ensure that individuals were aged $<18$ years during the entire period, age was assessed at the end of the panel for each subject. Insurance was categorized as private, public, or uninsured. Prescription drug coverage was coded as a binary variable if individuals were observed to have third-party payers in the MEPS "Prescribed Medicines" file. Family income level was stratified by the data source as a percentage of the federal poverty level (FPL): $<100,100-125,126-200,201-400$, and
$>400 \%$ of the FPL. Residence within a metropolitan statistical area (MSA) was recorded as a binary variable, and region was categorized by US census regions. Race was divided between non-Hispanic Whites, Hispanics (White or Black), African Americans (non-Hispanic), Asians, and others. Age, family income, region, MSA, and insurance type were all assessed at the last round of data collection.

Medical conditions were classified using single-level Clinical Classification System codes, which are based on valid International Classification of Disease, 9th Revision, Clinical Modification (ICD-9-CM) codes [11]. The number of prescriptions filled and number of unique medications filled over the 2-year panel were recorded as continuous measures.

\subsection{Data Analysis}

The proportion of LCGP uses was calculated as the proportion of all prescriptions filled via an LCGP in each year. The proportion of LCGP users was the percent of the population that filled at least one prescription via an LCGP. Comparisons were made between LCGP users and non-users in the 2011-2012 MEPS panel using chisquared or $t$ tests. Multivariable logistic regression was used to identify factors associated with LCGP use in the 2011-2012 panel. Adjusted odds ratios (AORs) and $95 \%$ confidence intervals (CIs) were reported. All data analyses were conducted using SAS 9.4 (SAS Institute, Cary, NC, USA) implementing SAS procedures (SURVEYMEANS, SURVEYFREQ, and SURVEYLOGISTIC) that take into account the complex survey design of MEPS and use the longitudinal survey weights supplied by MEPS to calculate population estimates over the 2 -year period. This manuscript was drafted according to the STROBE (Strengthening the Reporting of Observational Studies in Epidemiology) guidelines for observational research.

\section{Results}

\subsection{Cohort Comparison}

In the 2011-2012 MEPS panel, 2754 individuals were aged 0-17 years, participated in all rounds of data collection, and used at least one prescription medication during the study period. Applying MEPS person weights, this cohort represented a weighted population size of $43,020,913$ individuals who filled a prescription medication-over half of the US population aged $<18$ years (74.2 million) [12]. Of this population, $23.7 \%$ [95\% CI $21.1-26.34 \%$ ( $n=10,196,181)]$ were classified as LCGP users having 
filled at least one prescription meeting the aforementioned criteria for an LCGP fill.

User and non-user demographic characteristics are compared in Table 1. The LCGP user group included a significantly greater proportion of individuals who were White (71.3 vs. $57.4 \%)$, were in the highest family income level (34.0 vs. $21.7 \%$ ), had private insurance (83.1 vs. $54.8 \%$ ), were female (53.0 vs. $48.5 \%$ ), and lived in urban areas (87.2 vs. $84.3 \%)$. The LCGP non-user group had significantly more individuals with prescription drug coverage ( 83.8 vs. $68.7 \%$ ) than the user group. LCGP users tended to fill significantly more medications and used more unique medications than non-users. A significantly greater proportion of LCGP users experienced ear infections (33.6 vs. $23.2 \%$ ), respiratory infections (73.3 vs. $58.2 \%$ ), other general infections (24.5 vs. $15.9 \%)$, and mood disorders (7.1 vs. $2.1 \%$ ).

\subsection{Medication Use}

The study cohort $(n=2754)$ filled 20,739 prescriptions during the 2011-2012 panel period. Of the 20,739 medication fills, 66.1\% $(n=13,706)$ were for medications available through LCGPs. Of all fills for medications available through LCGPs, 8.6 \% were actually purchased through LCGPs. Figure 1 displays the proportions of LCGP fills and LCGP users graphed against the average total prescription fills per person per year in each year from 2007 to 2012. The proportion of LCGP fills out of all medications available through LCGPs decreased slightly from $8.2 \%$ of fills in 2007 to $8.1 \%$ of fills in 2012. Over 2007-2012, the proportion of LCGP users also decreased from $16 \%$ in 2007 to $14 \%$ in 2012 . While the proportions of LCGP fills and LCGP users both decreased slightly from 2007 to 2012, the number of prescription fills per person per year remained relatively stable at an approximate average of 3.4 fills per person per year.

Figure 2 shows the proportion of fills of each medication class that were purchased through LCGPs. The majority of metformin fills were obtained through LCGPs $(65.7 \%)$. Over $25 \%$ of fills for beta-blockers $(44.4 \%)$, ipratropium $(33.3 \%)$, diuretics $(31.8 \%)$, and analgesic combinations $(27.3 \%)$ were purchased through LCGPs. More than $15 \%$ of fills for levothyroxine (18.2\%), selective serotonin reuptake inhibitors (SSRIs) (18.0\%), tetracyclines $(17.9 \%)$, penicillins $(17.7 \%)$, topical anesthetics $(17.0 \%)$, contraceptives $(16.0 \%)$, and oral antiinfectives $(15.3 \%)$ were obtained through LCGPs. More than $10 \%$ of fills for topical anti-infectives (14.2\%), steroids $(13.4 \%)$, antifungals $(11.9 \%)$, topical acne medications (11.4\%), and tricyclic antidepressants (TCAs) $(10.8 \%)$ were filled through LCGPs. Other medication classes with fewer than $10 \%$ of fills through LCGPs included sulfamethoxazole/trimethoprim, topical steroids, fluoroquinolones, anti-emetics, urinary antispasmodics, topical antifungals, cephalosporins, angiotensin-converting enzyme inhibitors, anticonvulsants, and albuterol, among others.

\subsection{Determinants of LCGP Use}

After adjusting for all covariates, age, prescription drug coverage, insurance type, race, region, and the number of unique medications filled all significantly predicted LCGP use. The overall model c-statistic is 0.79 , reflecting strong discrimination between users and non-users. Results of the full regression model are presented in Table 2 .

Children aged 9-12 years were significantly less likely to use LCGPs than the reference category of children aged 0-4 years (AOR 0.59 [95\% CI 0.38-0.91]) and had lower odds than the groups aged 5-8 and 13-17 years. Individuals without prescription drug coverage were over $200 \%$ more likely than those with coverage to use LCGPs [95\% CI 2.35-4.52]. Publicly insured children (AOR 0.27 [95\% CI 0.18-0.39]) and those without any form of insurance (AOR 0.46 [95 \% CI 0.23-0.93]) were both significantly less likely to fill prescriptions through LCGPs. Asian children (AOR 0.44 [95 \% CI 0.20-0.99]) were also significantly less likely to fill medications through LCGPs than the White reference category. Individuals residing in the Midwest were less likely to use LCGPs than those living in the Northeast reference category (AOR 0.69 [95\% CI 0.48-0.99]). Each additional unique medication filled increased the odds of LCGP use by $33 \%$ (AOR 1.33 [95\% CI 1.25-1.41]).

\section{Discussion}

Our study found that nearly one in four people aged $<18$ years who used prescription drugs filled at least one medication through an LCGP during a 2-year period. Of all fills for medications available through LCGPs, $8.6 \%$ were purchased through LCGPs, or $5.7 \%$ of all medications were filled through LCGPs.

No known studies have assessed LCGP medication use in the pediatric and adolescent populations. One survey found that parents reported that their children with no insurance $(24 \%)$, public insurance $(23 \%)$, and private insurance (14\%) were using low-cost generics [9]. However, this survey did not investigate specific medication use in this population or consider other subject characteristics. Our study used data collected at the pharmacy level, which included specific payment sources, to determine program use. Applying MEPS person weights, we found that $32.0 \%$ of those with private insurance, $19.2 \%$ of those without 
Table 1 Characteristics of users and non-users of low-cost generic programs in the US pediatric population (age $<18$ years) during 2011-2012

\begin{tabular}{|c|c|c|c|c|c|c|c|c|}
\hline \multirow[t]{2}{*}{ Characteristic } & \multicolumn{4}{|c|}{ LCGP users } & \multicolumn{4}{|c|}{ Non-users } \\
\hline & $N$ & $\%$ & 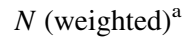 & $\%(\text { weighted })^{\mathrm{a}}$ & $N$ & $\%$ & 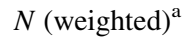 & $\%{\text { (weighted })^{\mathrm{a}}}$ \\
\hline Overall sample $(n=2754)$ & 525 & 19.1 & $10,196,181$ & 23.7 & 2229 & 80.9 & $32,824,732$ & 76.3 \\
\hline \multicolumn{9}{|l|}{ Age } \\
\hline $0-4$ & 147 & 28.0 & $2,924,435.00$ & 28.7 & 645 & 28.9 & $8,761,071$ & 26.7 \\
\hline $5-8$ & 131 & 25.0 & $2,320,994.00$ & 22.8 & 555 & 25.1 & $7,822,871$ & 23.8 \\
\hline $9-12$ & 102 & 19.4 & $1,858,311.00$ & 18.2 & 496 & 22.4 & $7,464,306$ & 22.7 \\
\hline $13-17$ & 145 & 27.6 & $3,092,442.00$ & 30.3 & 533 & 23.7 & $8,776,484$ & 26.7 \\
\hline \multicolumn{9}{|l|}{ Sex* } \\
\hline Male & 252 & 48.0 & $4,787,395$ & 47.0 & 1185 & 53.2 & $16,897,491$ & 51.5 \\
\hline Female & 273 & 52.0 & $5,408,786$ & 53.0 & 1044 & 46.8 & $15,927,241$ & 48.5 \\
\hline \multicolumn{9}{|l|}{ Insurance* } \\
\hline Private & 376 & 71.6 & $8,469,703$ & 83.1 & 846 & 38.0 & $17,976,449$ & 54.8 \\
\hline Public & 132 & 25.1 & $1,474,663$ & 14.5 & 1324 & 59.4 & $13,791,663$ & 42.0 \\
\hline Uninsured & 17 & 3.2 & 251,814 & 2.5 & 59 & 2.6 & $1,056,620$ & 3.2 \\
\hline \multicolumn{9}{|l|}{ Prescription coverage* } \\
\hline No coverage & 175 & 33.3 & $3,194,053$ & 31.3 & 280 & 12.6 & $5,313,686$ & 16.2 \\
\hline Coverage & 350 & 66.7 & $7,002,128$ & 68.7 & 1949 & 87.4 & $27,511,046$ & 83.8 \\
\hline \multicolumn{9}{|l|}{ Race* } \\
\hline Non-Hispanic White & 266 & 50.7 & $7,269,050$ & 71.3 & 775 & 34.8 & $18,829,880$ & 57.4 \\
\hline Hispanic (White or Black) & 148 & 28.2 & $1,615,182$ & 15.8 & 800 & 35.9 & $6,888,112$ & 21.0 \\
\hline African American & 68 & 13.0 & 793,037 & 7.8 & 474 & 21.3 & $4,641,232$ & 14.1 \\
\hline Asian & 22 & 4.2 & 240,551 & 2.4 & 81 & 3.6 & $1,196,827$ & 3.6 \\
\hline Other & 21 & 4.0 & 278,360 & 2.7 & 99 & 4.4 & $1,268,681$ & 3.9 \\
\hline \multicolumn{9}{|l|}{ Region* } \\
\hline Northeast & 73 & 13.9 & $1,561,263$ & 15.3 & 309 & 13.9 & $4,687,402$ & 14.3 \\
\hline Midwest & 107 & 20.4 & $2,226,796$ & 21.8 & 481 & 21.6 & $7,797,175$ & 23.8 \\
\hline South & 179 & 34.1 & $3,768,451$ & 37.0 & 873 & 39.2 & $13,388,854$ & 40.8 \\
\hline West & 166 & 31.6 & $2,639,671$ & 25.9 & 566 & 25.4 & $6,951,300$ & 21.2 \\
\hline \multicolumn{9}{|l|}{ Income category* } \\
\hline$<100 \%$ of FPL & 96 & 18.3 & $1,166,649$ & 11.4 & 875 & 39.3 & $8,581,904$ & 26.1 \\
\hline $100-125 \%$ of FPL & 20 & 3.8 & 308,487 & 3.0 & 173 & 7.8 & $1,937,629$ & 5.9 \\
\hline $125-200 \%$ of FPL & 88 & 16.8 & $1,507,038$ & 14.8 & 384 & 17.2 & $5,026,781$ & 15.3 \\
\hline $200-400 \%$ of FPL & 186 & 35.4 & $3,744,382$ & 36.7 & 503 & 22.6 & $10,164,330$ & 31.0 \\
\hline$>400 \%$ of FPL & 135 & 25.7 & $3,469,626$ & 34.0 & 294 & 13.2 & $7,114,087$ & 21.7 \\
\hline \multicolumn{9}{|l|}{ MSA* } \\
\hline Rural & 55 & 10.5 & $1,300,186$ & 12.8 & 321 & 14.4 & $5,161,161$ & 15.7 \\
\hline Urban & 470 & 89.5 & $8,895,995$ & 87.2 & 1908 & 85.6 & $27,663,570$ & 84.3 \\
\hline \multicolumn{9}{|l|}{ Conditions } \\
\hline Infections* & 121 & 23.0 & $2,501,823$ & 24.5 & 335 & 15.0 & $5,233,414$ & 15.9 \\
\hline Mental disorder & 74 & 14.1 & $1,597,277$ & 15.7 & 360 & 16.2 & $5,870,899$ & 17.9 \\
\hline ADHD & 46 & 8.8 & 832,986 & 8.2 & 248 & 11.1 & $3,933,646$ & 12.0 \\
\hline Mood disorder* & 32 & 6.1 & 728,963 & 7.1 & 52 & 2.3 & 692,575 & 2.1 \\
\hline Headache & 24 & 4.6 & 564,701 & 5.5 & 82 & 3.7 & $1,371,709$ & 4.2 \\
\hline Ear infection* & 166 & 31.6 & $3,422,828$ & 33.6 & 476 & 21.4 & $7,601,497$ & 23.2 \\
\hline Pneumonia & 28 & 5.3 & 667,777 & 6.5 & 77 & 3.5 & $1,255,095$ & 3.8 \\
\hline Respiratory infection* & 365 & 69.5 & $7,475,498$ & 73.3 & 1229 & 55.1 & $19,117,431$ & 58.2 \\
\hline Asthma & 95 & 18.1 & $1,518,811$ & 14.9 & 387 & 17.4 & $5,257,033$ & 16.0 \\
\hline Arthritis & 43 & 8.2 & 971,159 & 9.5 & 148 & 6.6 & $2,353,757$ & 7.2 \\
\hline
\end{tabular}


Table 1 continued

\begin{tabular}{|c|c|c|c|c|c|c|c|c|}
\hline \multirow[t]{2}{*}{ Characteristic } & \multicolumn{4}{|c|}{ LCGP users } & \multicolumn{4}{|c|}{ Non-users } \\
\hline & $N$ & $\%$ & 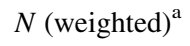 & $\%{\text { (weighted })^{\mathrm{a}}}$ & $N$ & $\%$ & 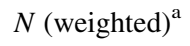 & $\%(\text { weighted })^{\mathrm{a}}$ \\
\hline Diabetes & 8 & 1.5 & 135,413 & 0.5 & 9 & 0.4 & 151,559 & 0.5 \\
\hline Nutritional deficiencies* & 12 & 1.7 & 242,186 & 2.4 & 41 & 1.8 & 198,243 & 0.6 \\
\hline *Epilepsy & 9 & 2.3 & 229,048 & 2.2 & 13 & 0.6 & 472,714 & 1.4 \\
\hline Total number of medication fills* (median [IQR]) & \multicolumn{2}{|c|}{$4(2-10)$} & \multicolumn{2}{|l|}{$4(2-10)$} & \multicolumn{2}{|c|}{$3(1-7)$} & \multicolumn{2}{|l|}{$2(1-7)$} \\
\hline Unique medications used* (median [IQR]) & \multicolumn{2}{|c|}{$3(2-5)$} & \multicolumn{2}{|l|}{$2(1-4)$} & \multicolumn{2}{|c|}{$2(1-3)$} & $1(1-3)$ & \\
\hline
\end{tabular}

Percentages not adding to $100 \%$ are due to rounding

$A D H D$ attention-deficit hyperactivity disorder, FPL federal poverty limit, IQR interquartile range, LCGP low-cost generic program, MSA metropolitan statistical area

${ }^{a}$ MEPS data are weighted based on demographics (e.g., race, sex, age, etc.) to be nationally representative. The total raw sample included 2754 subjects who, when weighted, represent $43,020,913$ subjects in the USA

$* p<0.05$ for weighted group comparison of LCGP users vs. non-users on all characteristics

Fig. 1 Trend of the proportion of low-cost generic program (LCGP) users and fills and overall medication utilization in each year 2007-2012.

Proportion of users is the proportion of the pediatric population that filled at least one prescription through an LCGP in that year. Proportion of fills is the proportion of prescription fills obtained via an LCGP out of all prescription fills that are available through LCGPs in the pediatric population for that year

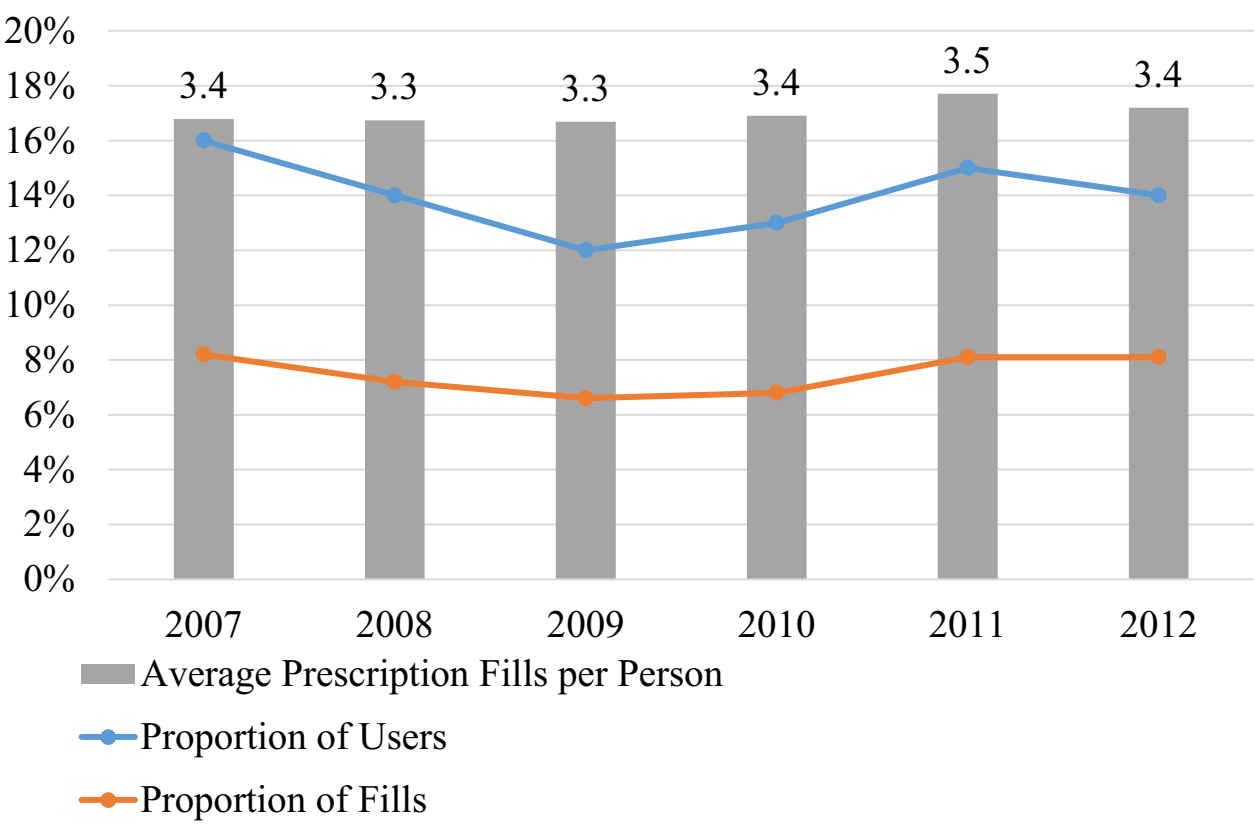

insurance, and only $9.7 \%$ of those with public insurance used LCGPs (Table 1). In addition to these differences, the overall proportion of users in our study (23.7\%) was slightly higher than the prevalence of use determined in the prior study (18\%) [9].

Children who lack any form of insurance or prescription drug coverage may stand to benefit the most from the inexpensive medications offered through LCGPs. Given the findings that lack of prescription drug coverage is a significant predictor of LCGP use (AOR 3.26 [95\% CI 2.35-4.52]; Table 2), and that nearly one in five uninsured children used an LCGP from 2011 to 2012 (Table 1), it can be seen that this population is indeed using these programs. However, in adjusted analysis, the uninsured pediatric population had roughly $50 \%$ lower odds of using LCGP medications than privately insured children (Table 2). Furthermore, the finding that most LCGP users fall into the two highest income levels suggests that individuals who could benefit most from these programs are not in fact using them as often as they could, suggesting differential access to care among insurance and family income groups $[13,14]$.

Previous studies have reported cost and access to medications as significant barriers to proper management of 
Fig. 2 Percentage of each medication class filled through low-cost generic program (LCGPs) during 2011-2012. $A C E I$ angiotensin-converting enzyme inhibitor, NSAID nonsteroidal anti-inflammatory drug, $S M X-T M P$ sulfamethoxazoletrimethoprim, SSRI selective serotonin reuptake inhibitor, $T C A$ tricyclic antidepressant

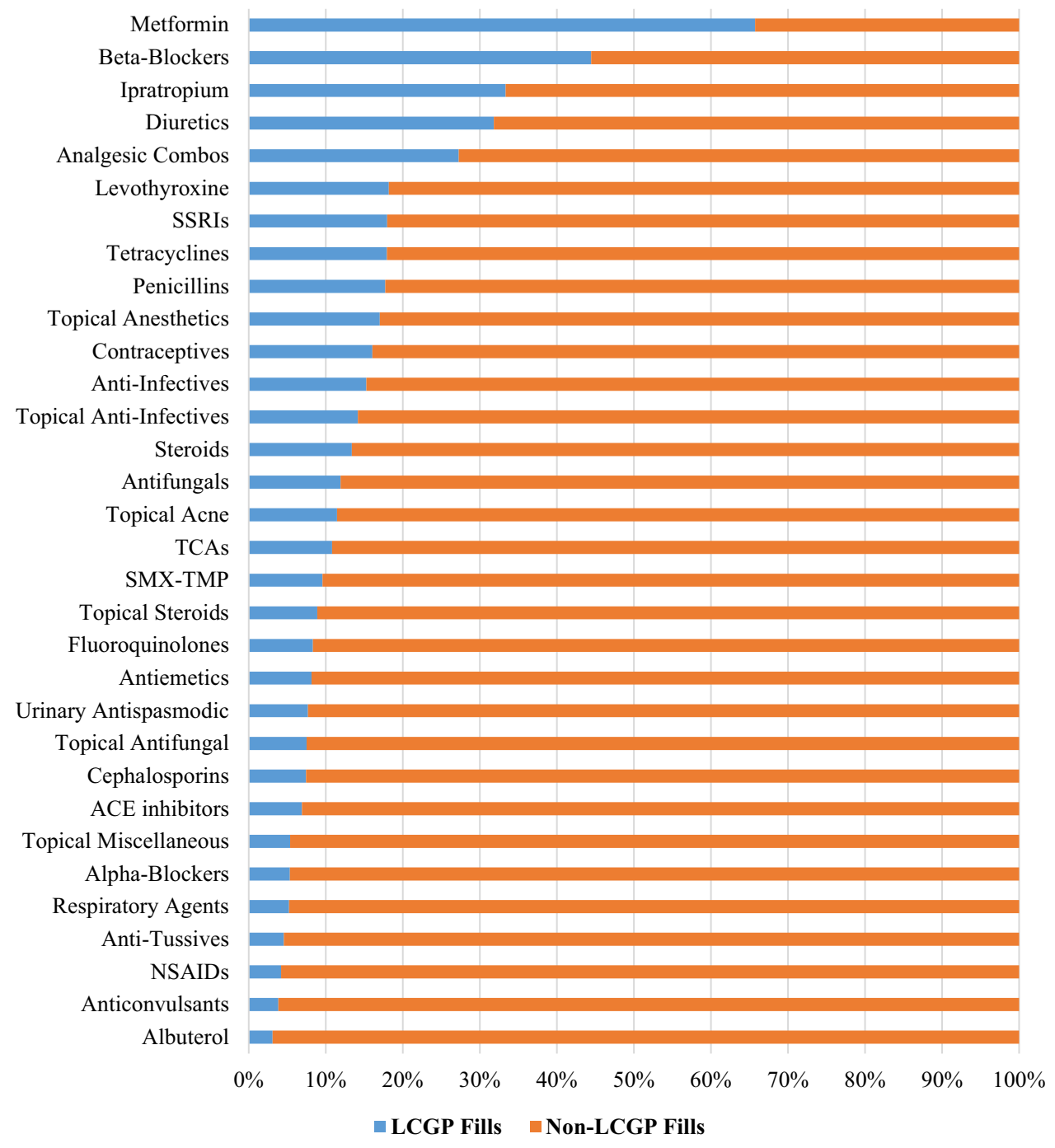

pediatric conditions [15-17]. Inexpensive medications offered through LCGPs improve access to and ameliorate the cost burden of prescription medications for children across the USA. Several findings of this study indicate that LCGPs are commonly being used to assist with the management of both chronic and acute illnesses. Results of this study indicate that rates of LCGP use in the 2011-2012 MEPS panel are markedly higher in children with diseases that can be treated with medications available through LCGPs than the rate in the overall pediatric population $(23.7 \%)$. For example, $55.0 \%$ of children with nutritional deficiencies were LCGP users, as well as $47.2 \%$ of those with diabetes, $34.7 \%$ of those with pneumonia, $32.6 \%$ of those with epilepsy, $32.3 \%$ of those with general infections, $31.0 \%$ of those with ear infections, $29.2 \%$ of children with arthritis, and $28.1 \%$ of those with respiratory infections (Table 1).

Despite the benefits of greater LCGP use in the pediatric population, astute researchers must be wary of the potential for exposure misclassification that these programs present when using certain data sources. True medication utilization may not be captured in the administrative claims for individuals who use LCGPs, which may impact the findings of health services research relying on these data sources [18, 19]. Medication use in this age group includes medication classes with significant risk profiles and medications used to treat serious medical conditions. Especially for privately insured children, who according to our findings use LCGPs at a greater rate than publicly insured or uninsured children, exposure misclassification due to LCGP use may circumvent the added protections (i.e., surveillance for drug interactions, etc.) provided by the claims adjudication process. For children with public forms of insurance, exposure misclassification due to LCGP use may have important ramifications on the calculation of quality metrics that Medicaid plans are required to report under the ACA [20, 21]. Using Fig. 2, the potential impact of misclassification can be estimated given that the 
Table 2 Multivariable logistic regression results of predictive characteristics for LCGP use in the United States pediatric population in 2011-2012

\begin{tabular}{|c|c|c|c|}
\hline \multirow[t]{2}{*}{ Characteristic } & \multirow[t]{2}{*}{ Adjusted OR } & \multicolumn{2}{|c|}{$95 \%$ wald confidence limits } \\
\hline & & Lower & Upper \\
\hline \multicolumn{4}{|l|}{ Age } \\
\hline $0-4$ & Ref. & Ref. & Ref. \\
\hline $5-8$ & 0.86 & 0.58 & 1.28 \\
\hline $9-12$ & 0.59 & 0.38 & 0.91 \\
\hline $13-17$ & 0.78 & 0.55 & 1.10 \\
\hline \multicolumn{4}{|l|}{ Sex } \\
\hline Male & Ref. & Ref. & Ref. \\
\hline Female & 1.13 & 0.88 & 1.45 \\
\hline \multicolumn{4}{|l|}{ Prescription drug coverage } \\
\hline Prescription coverage & Ref. & Ref. & Ref. \\
\hline No prescription coverage & 3.26 & 2.35 & 4.52 \\
\hline \multicolumn{4}{|l|}{ Insurance type } \\
\hline Private & Ref. & Ref. & Ref. \\
\hline Public & 0.27 & 0.18 & 0.39 \\
\hline Uninsured & 0.46 & 0.23 & 0.93 \\
\hline \multicolumn{4}{|l|}{ Income category } \\
\hline$<100 \%$ of FPL & Ref. & Ref. & Ref. \\
\hline $100-125 \%$ of FPL & 1.02 & 0.50 & 2.10 \\
\hline $125-200 \%$ of FPL & 1.41 & 0.90 & 2.19 \\
\hline $200-400 \%$ of FPL & 1.29 & 0.83 & 2.02 \\
\hline$>400 \%$ of FPL & 1.46 & 0.89 & 2.39 \\
\hline \multicolumn{4}{|l|}{ Race } \\
\hline Non-Hispanic White & Ref. & Ref. & Ref. \\
\hline Hispanic (White or Black) & 1.02 & 0.71 & 1.46 \\
\hline African American (non-Hispanic) & 0.70 & 0.45 & 1.10 \\
\hline Asian & 0.44 & 0.20 & 0.99 \\
\hline Other & 0.67 & 0.37 & 1.21 \\
\hline \multicolumn{4}{|l|}{ MSA } \\
\hline Rural & Ref. & Ref. & Ref. \\
\hline Urban & 1.17 & 0.76 & 1.82 \\
\hline \multicolumn{4}{|l|}{ Region } \\
\hline Northeast & Ref. & Ref. & Ref. \\
\hline Midwest & 0.69 & 0.48 & 0.99 \\
\hline South & 0.82 & 0.55 & 1.21 \\
\hline West & 1.17 & 0.79 & 1.72 \\
\hline Number of unique meds & 1.33 & 1.25 & 1.41 \\
\hline
\end{tabular}

$F P L$ federal poverty level, $L C G P$ low-cost generic program, $M S A$ metropolitan statistical area proportion of fills of each medication class filled through an LCGP provides an estimate of the number of exposures that may go unclassified.

Our study is subject to some limitations. It may remain possible that not all medication use is recorded if all pharmacies used were not surveyed. Our study definition of LCGP use may allow for overestimation of use if only pricing is considered. However, this effect is mitigated by requiring specific quantities supplied for oral medications. Finally, it is possible that some individuals exclusively use pharmacies in which LCGPs are not available and thus this population was never eligible for inclusion in the LCGP user cohort.

\section{Conclusions}

This study found a high rate of LCGP users among a nationally representative sample of children and adolescents. Low-cost generics increase the affordability and 
access to medications where high costs may prohibit treatment. However, considering the implications for patients and the healthcare system as a whole, programs should be implemented so that medication use data are captured and are accounted for in drug policy and pharmacoepidemiological research. Future studies should empirically demonstrate the effects of differential exposure misclassification on the results of claims-based research.

Author contributions All study authors participated in the design, analysis, interpretation, and drafting of the manuscript, meet the criteria for authorship, and approve of the final version of this manuscript.

\section{Compliance with Ethical Standards}

Ethical approval The MEPS is a publicly available, de-identified data source collected for research purposes; thus, it is considered exempt by the University of Kentucky Institutional Review Board.

Conflict of interest Joshua Brown receives salary support from both Humana and Pfizer; however, neither organization was involved with any aspect of this study, including design, analysis, or drafting of the manuscript, nor provided any direct funding for the conduct of the study. There are no other conflicts of interest to disclose for this work for Nathan Pauly or Jeffery Talbert.

Funding No sources of funding were used to assist in the preparation of this study.

Open Access This article is distributed under the terms of the Creative Commons Attribution-NonCommercial 4.0 International License (http://creativecommons.org/licenses/by-nc/4.0/), which permits any noncommercial use, distribution, and reproduction in any medium, provided you give appropriate credit to the original author(s) and the source, provide a link to the Creative Commons license, and indicate if changes were made.

\section{References}

1. Czechowski JL, Tjia J, Triller DM. Deeply discounted medications: implications of generic prescription drug wars. J Am Pharm Assoc. 2010;50:752-7. doi:10.1331/Japha.2010.09114.

2. Choudhry NK, Shrank WH. Four-dollar generics-increased accessibility, impaired quality assurance. N Engl J Med. 2010;363:1885-7. doi:10.1056/Nejmp1006189.

3. Patel HK, Dwibedi N, Omojasola A, Sansgiry SS. Impact of generic drug discount programs on managed care organizations. Am J Pharm Benefits. 2011;3(1):45-53.

4. Walmart. Walmart retail prescription program drug list. 2015. http://i.walmartimages.com/i/if/hmp/fusion/customer_list. pdf. Accessed 25 Mar 2015.
5. Club Wps. Value-priced medication list. 2014. http://www. walgreens.com/images/psc/VPG_List_Update_01-02-2015.pdf. Accessed 25 Mar 2015.

6. Nl Rucker. \$4 generics: How low, how broad, and why patient engagement is priceless. J Am Pharm Assoc. 2010;50:761-3. doi:10.1331/Japha.2010.10546.

7. Tungol A, Starner CI, Gunderson BW, Schafer JA, Qiu Y, Gleason PP. Generic drug discount programs: are prescriptions being submitted for pharmacy benefit adjudication? J Manag Care Pharm Jmcp. 2012;18:690-700.

8. Zhang Y, Zhou L, Gellad WF. Potential savings from greater use of $\$ 4$ generic drugs. Arch Intern Med. 2011;171:468-9. doi:10. 1001/Archinternmed.2011.46.

9. Hospital Csmcs. Nearly 70 million americans using discount generic Rx programs. 2008. http://mottnpch.org/sites/default/files/ documents/021108GenericRxPrograms.pdf. Accessed 25 Mar 2015.

10. An Hofer, Jm Abraham, Ira Moscovice. Expansion of coverage under the patient protection and affordable care act and primary care utilization. Milbank Q. 2011;89(1):69-89. doi:10.1111/J. 1468-0009.2011.00620.X.

11. (Hcup) Hcaup. Hcup Ccs. Agency for healthcare research and quality, Rockville, Md. 2015. http://www.hcup-us.ahrq.gov/ toolssoftware/ccs/ccs.jsp. Accessed 16 Jun 2015.

12. Howden LM, Meyer JA. Age and sex composition: 2010: US Census Bureau 2011; May 2011.

13. Heisler M, Wagner TH, Piette JD. Patient strategies to cope with high prescription medication costs: who is cutting back on necessities, increasing debt, or underusing medications? J Behav Med. 2005;28(1):43-51.

14. Le Felland, Reschovsky Jd. More nonelderly americans face problems affording prescription drugs. Track Rep. 2009;22:1-4.

15. Halfon N, Newacheck PW. Childhood asthma and poverty: differential impacts and utilization of health services. Pediatrics. 1993;91(1):56-61.

16. Lm Ingerski, Rn Baldassano, La Denson, Ka Hommel. Barriers to oral medication adherence for adolescents with inflammatory bowel disease. J Pediatr Psychol. 2010;35(6):683-91. doi:10. 1093/jpepsy/jsp085.

17. Pelaez S, Aj Lamontagne, Collin J, Gauthier A, Rm Grad, Blais L, et al. Patients' perspective of barriers and facilitators to taking longterm controller medication for asthma: a novel taxonomy. BMC Pulm Med. 2015;15(1):42. doi:10.1186/S12890-015-0044-9.

18. Jacobus S, Schneeweiss S, Chan KA. Exposure misclassification as a result of free sample drug utilization in automated claims databases and its effect on a pharmacoepidemiology study of selective cox-2 inhibitors. Pharmacoepidemiol Drug Safety. 2004;13:695-702. doi:10.1002/pds.981.

19. Polinski JM, Schneeweiss S, Levin R, Shrank WH. Completeness of retail pharmacy claims data: implications for pharmacoepidemiologic studies and pharmacy practice in elderly adults. Clin Ther. 2009;2009(31):2048-59. doi:10.1016/j.clinthera.2009.09.009.

20. Assurance Ncfq. Hedis ${ }^{\circledR}$ and quality compass ${ }^{\circledR}$. http://www.ncqa. org/HEDISQualityMeasurement/WhatisHEDIS.aspx. Accessed 25 Mar 2015.

21. Implementation of section 2717: ensuring the quality of care. National Committee for Quality Assurance. 